\title{
VISION ALGORITHM FOR SEAM TRACKING IN Automatic Welding System
}

\author{
Arun Prakash ${ }^{1}$ \\ ${ }^{1}$ Assistant Professor, Department of Mechanical Engineering, SSN College of \\ Engineering, Chennai, India
}

\begin{abstract}
Arc welding is an important technology for joining metals and repairing metal products in manufacturing industries. It is usually performed by a skilled worker. Due to the shortage of skilled workers and due to the hazardous and unpleasant working conditions, the automation of arc welding is gaining importance nowadays. Existing welding robots require that the dimensions, position and orientation to be programmed prior to the process. These robots suffer from an evident lack of flexibility. This paper presents an automatic welding system assisted by a machine vision system to compute the dimension, position and orientation of the workpieces. By analysing the image taken by the camera, a simple algorithm is developed to detect the dimension, position and orientation of the workpieces. The calculated data is then fed to a controller which controls the welding electrode movement. Use of machine vision system has eliminated the need to pre feed the workpiece data to the robot and hence the system is made flexible.
\end{abstract}

\section{KEYWORDS}

Machine Vision, Automatic Welding System, Robotic Welding, Image Processing, Arc Welding

\section{INTRODUCTION}

Welding is defined as the process of joining similar metals by the application of heat. During welding, the edges of the metal pieces are either melted or brought to plastic condition. This process is used for making permanent joints, which is obtained by homogenous mixture of two materials. Now a days, welding finds wide spread applications in almost all branches of engineering industry. It is extensively employed in the fabrication and erection of steel structure in industries and construction [1]. It is also used in various industries like aircraft frame works, railway wagons, furniture, automobile bodies, ship buildings, nuclear industries etc. depending on their application [2]. Arc welding is usually performed by a skilled human worker who is often assisted by a person called fitter. The working conditions of the welder are typically unpleasant. The arc from the welding process emits ultraviolet radiation which is injurious to human vision. Other aspects of the process are also hazardous. For instance, the high temperatures created in arc welding and the resulting molten metal is inherently dangerous. The electrical current that is used to create the welding arc is also unsafe. During the welding process, sparks and smoke are generated and these make the environment unsafe to the operator. As a result of these difficulties, robots are being employed on the production line to perform arc welding operations. The robot is programmed to perform a sequence of welding operation on the product as it arrives at the workstation [3]. However, there are significant technical and economic problems encountered in applying robots to arc welding. One of the most difficult technical problems for welding robots is the presence of variations in the components that are to be welded [4]. One is the variation in the dimensions of the parts in a batch production job. This type of dimensional variation means that the arc welding path to be followed will slightly change from part to part. The second variation is in the position of orientation of workpiece itself. These two problems can be overcome if we opt for a machine vision system. The machine vision system consists of a camera and a controller. 
The camera takes the image of the worktable with workpiece on it and the controller computes the dimension, position and orientation (horizontal or vertical) of the workpiece. Based on the calculations, the controller controls the electrode position and the welding can be done accurately irrespective of the orientation and position of the workpieces.

\section{Automation OF WeLdiNG}

This is an era of automation where automation is broadly defined as replacement of manual effort by mechanical power. Automatic welding system can be classified into two categories namely:

- System based on contact sensors

- System based on noncontact sensors

This paper focuses on the latter. There are three categories of non contact sensors: [5]

i. Systems based on voltage through the arc welding

ii. $\quad$ Systems based on ultrasonic sensors

iii. Systems based on visual sensors

Beningo Maqueria, et al(1989) [6] developed an ultrasonic based robot to track the seam. He used an ultrasonic sensor which is interfaced to a P-50 process robot in an effort to achieve on-line seam tracking of joints, without the use of geometrical models or subsequent "teaching" routines. Ai-min Li, et al (2011) [7] designed an automatic welding machine based on PLC. He designed the machine to perform carbon-di-oxide gas welding.

\section{MACHINE VISION}

\subsection{Anatomy of Machine Vision}

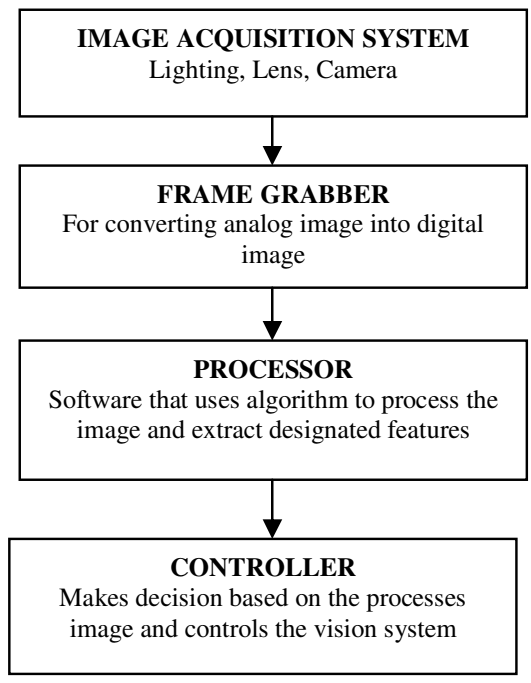

Figure 1. Anatomy of machine vision

Machine vision can be defined as the interpretation of an image of an object or scene through the use of optical non contact sensing mechanics for the purpose of obtaining information and/or 
controlling machines or processes. The machine vision system consists of an image acquisition system, frame grabber, image processing software and a controller. The anatomy of a simple machine vision system is shown in Figure 1. The image acquisition system comprises of lighting system, lens and a camera. The efficiency of a machine vision system mainly depends on this image acquisition system. Once a proper image is acquired, then the remaining steps of the image processing become quite simpler. The frame grabber is used to convert an analog image into a digital image. It has to be used only if image acquisition device is an analog camera. The image processing software is used to execute the image processing algorithm and extract the designated features from the image. The controller is used to take decisions and/or control the system based on the output from the image processing software. In the automatic welding system the controller controls the actuator to which the electrode is attached.

\subsection{Machine Vision in Welding}

Now-a-days, machine vision has a tremendous scope in the shop floor, not only in the quality control but also in the manufacturing field, especially to enhance the utilization of automation using robots. The integration of computer vision with robot motion is steadily progressed by adding the visual feedback. The increasing demand of the welding process in the production shop heavily depends on the skilled operator. But it is very difficult to find these operators or maintain them in an industry. Though humans are superior to machines in many ways, they too have some disadvantages such as suffering from fatigue, definite working hours etc. Also it is necessary to complete the weld task at places where it is difficult for human to go such as, the weld task in the outer space, underwater, and nuclear environment. In such cases we go for robotic welding based on vision. Machine vision has many advantages such as efficiency and high reliability. These systems are highly consistent and they can also measure dimensions within thousandths-of-aninch accuracy. The block diagram of a vision based welding diagram is shown in Figure 2.

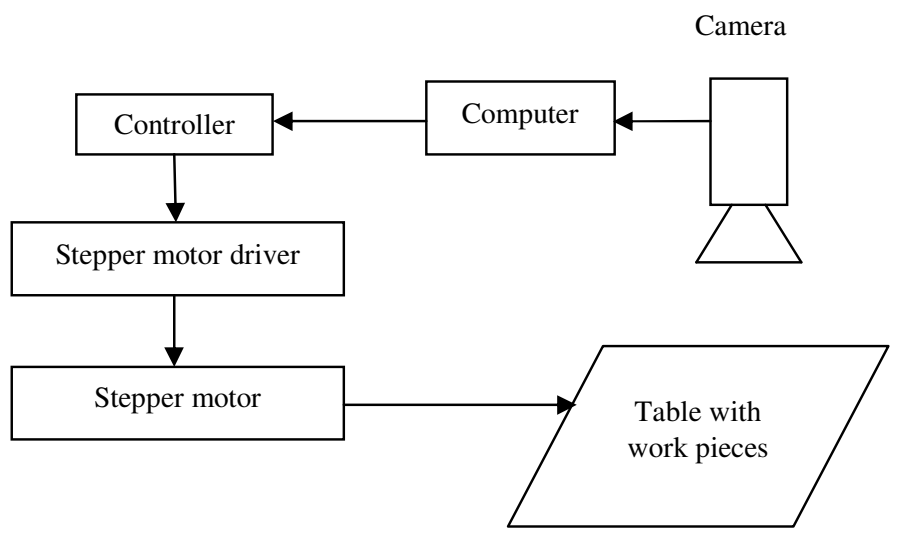

Figure 2. Block diagram of vision based welding system

Mamoru Kawahara, et al (1983) [8] developed a tracking system using image sensor for arc welding. The control system uses servo mechanism, a solid state image sensor and an image processor. The image processor consisting of microcomputer, processes video information and estimates the position of the welding groove centre using a statistical technique.

Dukun Ding, et al (2009) [2] developed a weld pool image centroid algorithm for seam tracking in arc welding process. The weld pool images are captured by a camera arranged ahead of a welding torch. Image processing techniques are employed to analyze the features of the weld pool 
and its surroundings and the centroid of weld pool image is extracted as a parameter for seam tracking.

Alberto Fernandez, et al (2011) [9] developed a low cost system for the weld tracking based on artificial vision in which the laser is setup perpendicular to the workpiece surface with the webcam on the robot arm oriented towards the projected laser stripe at an angle of $45^{\circ}$. The software tracks the deepest point in the welding seam using algorithms and positions the welding head above it.

In all the above mentioned systems, the camera is attached to the arm whereas in this paper we discuss a setup where the camera is attached separately and not on the arm.

\section{HARDWARE SETUP}

\subsection{Mechanical Design}

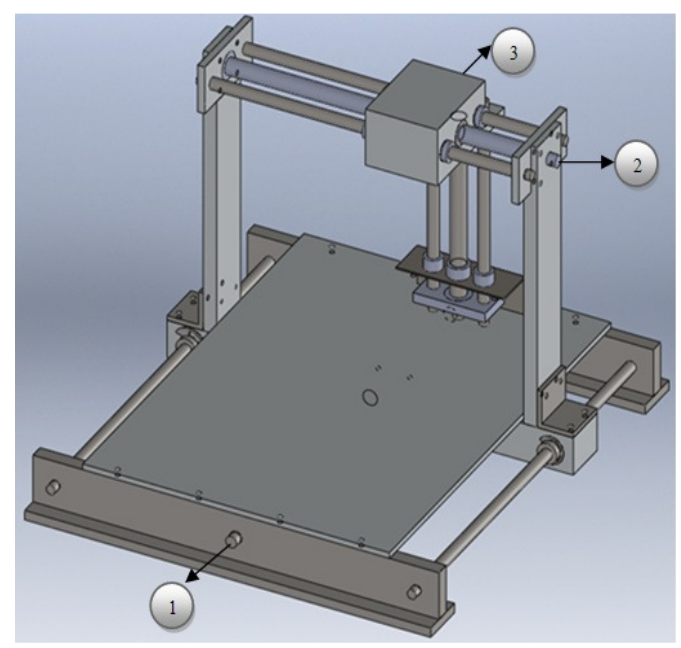

Figure 3. CAD model of XYZ table

The mechanical design of the automatic welding machine with the position of motors indicated as $1,2,3$ is shown in Figure 3. The mechanical setup consists of a XYZ table with stepper motors attached to it for the movement of the electrode in the required direction. The base plate has a dimension of $465 \times 340 \times 6 \mathrm{~mm}$. The plates that are to be welded are placed on the top of the base plate. Three lead screws are used for the $\mathrm{X}, \mathrm{Y}$ and $\mathrm{Z}$ axes movement and the specifications of the lead screws are as follows: the lead screw for X-Axis has a length of $510 \mathrm{~mm}$, dia of $16 \mathrm{~mm}$ and pitch of $4 \mathrm{~mm}$; the lead screw for Y-Axis has a length of $415 \mathrm{~mm}$, dia of $16 \mathrm{~mm}$ and pitch of $4 \mathrm{~mm}$; the lead screw for Z-Axis has a length of $265 \mathrm{~mm}$, dia of $16 \mathrm{~mm}$ and pitch of $4 \mathrm{~mm}$.

Stepper motor placed at the base position (indicated as 1 in Figure 3 ) is used to move the arm in the $\mathrm{X}$ direction. As it has to carry the entire weight of the setup a high torque stepper motor is chosen. The stepper motor placed in the position 2 as indicated in Figure 3 is used for movement of the arm in $\mathrm{Y}$ direction. It carries a block on which the lead screw and the guide rods for $\mathrm{Z}$ axis movement are mounted. The stepper motor placed in the position 3 as indicated in Figure 3 is used for the electrode movement in the $\mathrm{Z}$ direction. The speed of the $\mathrm{Z}$ axis motor and the pitch of the lead screw determine the feed rate of the electrode. The feed rate of the electrode varies with the type of material and the type of electrode. Thus the speed of the motor and the pitch of the 
lead screw are determined by the type of the material that is welded and the consumption rate of the electrode. Three limit switches are fixed on the table one for each axis. Limit switches are used to ensure that the electrode is in initial position during the beginning of the process.

\subsection{Vision System}

A camera is used to record the image of the workpieces placed on the table. Area scan camera has to be used as the image of entire worktable has to be recorded. AVT stingray camera of resolution $1624(\mathrm{H}) \mathrm{X} 1234(\mathrm{~V})$ is used in this experiment.

\subsection{Control System}

The automatic welding machine uses microcontroller to control and actuate the stepper motors. By controlling the stepper motors, the electrode motion can be guided. The input to the microcontroller is received from the PC and limit switches and the output of the microcontroller is sent to the stepper motors.

\section{WORKING OF THE SYSTEM}

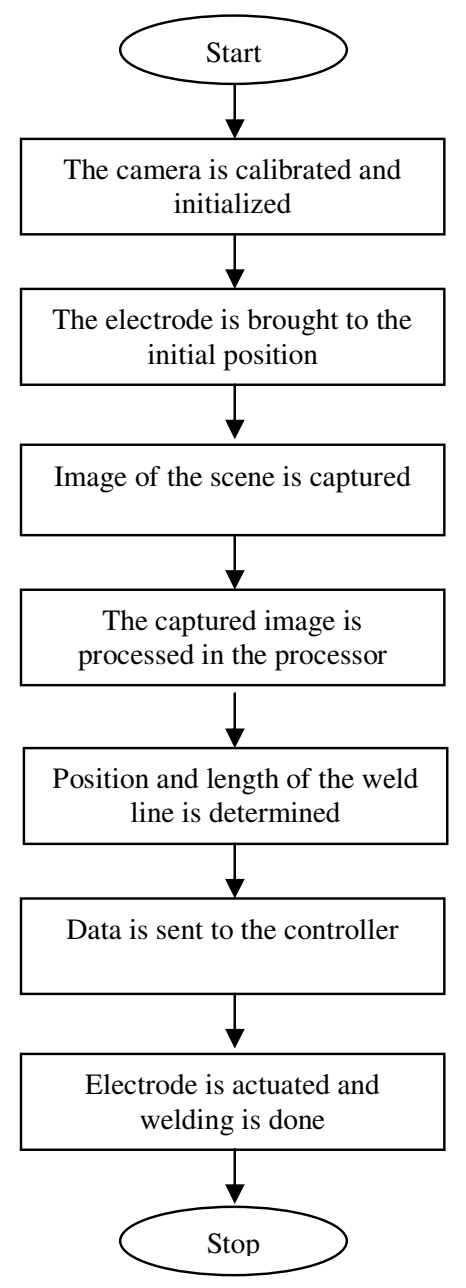

Figure 4. Workflow of the system 
Camera calibration is done before starting the working process. The output from the limit switch is checked by the microcontroller to ensure that the electrode is in the initial position before the process commences. After bringing the electrode to the initial position, the image processing algorithm is executed.

The image of the worktable with workpieces on it is captured using a camera. The image is processed in the processor and the weld line along which the welding has to be performed is detected. The position, orientation (horizontal or vertical) and the dimension of the weld line are calculated by the processor which is then sent to the controller through the serial port. The electrode is then guided by the controller to move along the weld line by actuating the stepper motor. As the electrode moves along the weld line, welding is performed throughout the calculated length of the workpiece. Once the welding is completed, the electrode is brought back to the initial position. The flowchart of the process is shown in Figure 4.

\section{Image Processing Algorithm}

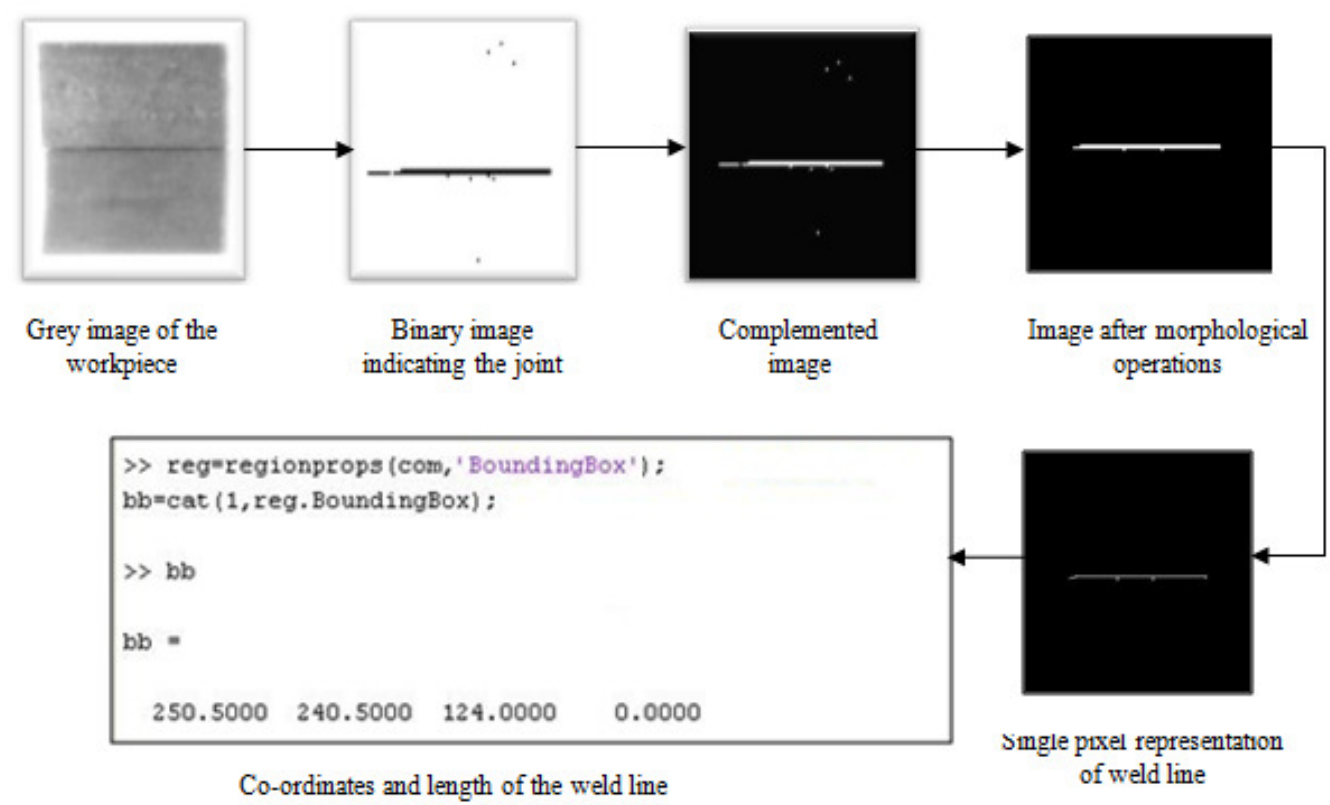

Figure 5. Various images obtained during processing

Before starting the image processing algorithm, it is necessary to calibrate the camera. In general, camera calibration is the problem of determining the intrinsic and extrinsic parameters of a camera. It involves mapping the real world co-ordinates to the camera sensor plane co-ordinates. After calibrating the camera, the image of the workpieces placed on the worktable is captured. The captured image is then converted into a binary image (image with only black and white pixels) by selecting a proper threshold value. The threshold value is chosen in such a way that the weld line is converted into black pixels and all other parts of the image are converted into white pixels. The image is then complemented during which the black pixels are converted into white and vice versa. Morphological operations are performed to remove the noise (unwanted regions) in the image. Skeleton of the image is then obtained so as to reduce the weld line to single pixel thickness. Then the pixel information are gathered and then fed to the controller in order to control the electrode. The image conversions that take place during processing are shown in the Figure 5 . 
The first two bounding box values $(250.5,240.5)$ as shown in Figure 5 indicate the starting coordinates of the weld line and the third value (124) indicates the length of the weld line. The fourth value which is zero indicates that the weld line is horizontal. If the weld line is vertical, then the third value becomes zero and the fourth value indicates the length of the weld line. All the values are in terms of the pixel coordinates. They are converted to world through camera calibrations. Experiment has shown that dividing the pixel coordinate values with 20 gives the coordinates in centimetres. Thus in the above data the length of the weld line is $6.2 \mathrm{~cm}$.

\section{CONCLUSION}

In this paper, a vision algorithm using $2 \mathrm{D}$ image processing techniques to automate welding was proposed and tested. As image processing is used, the system becomes flexible adapting itself to the changes in the position and dimensions of the workpiece. This algorithm, when implemented in an automatic welding system, makes it simpler and effective in improving the quality of the weld and also increases the welding efficiency. It also significantly reduces the labour intensity with good economic returns.

\section{REFERENCES}

[1] M.A. Wahab, "Manual Metal Arc Welding and Gas Metal Arc Welding", Comprehensive Materials Procesing, Vol 6, 2014, Pages 49-76

[2] Xiangdong Gao, Dukun Ding, Tianxiang Bai, "Weld Pool Image Centroid Algorithm for Seam Tracking in Arc Welding Process", International Workshop on Imaging Systems and Techniques", 2009.

[3] Pritschow. G.Muller. S, Horber. H, "Fast and robust image processing for laser stripe-sensors in arc welding automation", Proceedings of International Symposium of Industrial Electronics, Vol 2, 2002.

[4] Mikell P.Groover, Mitchell Weiss, Roger N.Nagel, "Industrial Robotics-Technology, Programming and Applications".

[5] Alberto Fernandez, Antonio Lopez, Jose Manuel Garcia,” Low-Cost System for Weld Tracking Based on Artificial Vision", IEEE Transactions On Industry Applications, Vol. 47, No. 3, May/June 2011

[6] Beningo, Charles and Jacek, "Application of Ultrasonic sensors to Robotic Seam Tracking", IEEE Transactions on Robotics and Automation, Vol.5, 1989

[7] Ai-min Li, Hai-lin Li, Sheng-wei Ye, "Design of Automatic Welding Machine Based on PLC", Fourth International Conference on Intelligent Computation Technology and Automation, 2011

[8] Mamoru Kawahara, "Tracking Control System Using Image Sensor for Arc Welding”, Automatica, Vol.19,No.4, 1983.

[9] Alberto Fernandez, Antonio Lopez, Jose Manuel Garcia," Low-Cost System for Weld Tracking Based on Artificial Vision", IEEE Transactions On Industry Applications, Vol. 47, No. 3, May/June 2011

\section{Author}

Arun Prakash. C obtained his M.E from Anna University, M.I.T Campus Chennai.He is currently working as an Assistant Professor in the department of Mechanical Engineering at Sri Sivasubramaniya Nadar (SSN) College of Engineering, Chennai

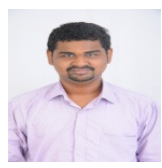

